\title{
A Survey on the Internet-of-Things: Standards, Challenges and Future Prospects
}

\author{
KATSUHIRO NaITo ${ }^{1, a)}$ \\ Received: September 6, 2016, Accepted: November 7, 2016
}

\begin{abstract}
Technological development in communications and electronics has made the growing expansion of the Internet of Things (IoT). IoT is expected to make a great impact to our society because smart devices in IoT are easily integrated into existing service. As a result, standardization of technologies to support the IoT is becoming more important to realize a smart society through different service domains. This paper presents a survey on the current state of the art of standards for IoT technologies and gives a brief introduction to related standards and recent research areas in IoT. Finally, it also proposes an idea of the future platform of scalable IoT systems. The proposed idea employs IP mobility technologies to realize inter-operability among IoT devices in different networks.
\end{abstract}

Keywords: IoT, M2M, protocol, standard, IP mobility

\section{Introduction}

The Internet of Things (IoT) is a new technology that is gaining ground due to the huge advancements in the fields of electronics and wireless communication technologies [1]. The concept of the IoT is collecting various information and analyzing it for application services. Therefore, the basic idea of IoT is almost same in Cyber Physical Systems (CPS) [2]. CPS consists of physical systems such as sensor networks for data collecting and cyber systems for sophisticated data-analysis.

CPSs have three special constraints: a limited resource, special-purpose design and a functional interaction. Firstly, typical devices in CPS have limited memory, computing capability and energy, which prevent the use of conventional full stack IP protocols [3]. Therefore, resource constrained protocols such as 6LoWPAN [4] have been considered. Secondary, CPSs are designed for special applications related to the physical phenomenon. Hence, almost all routing protocols for fixed sensor networks are insufficient in practical usages and new protocols should be designed for CPSs. Thirdly, decentralized and distributed control loops require data and control interfaces. However, general interfaces may not be appropriate to realize a special application because special applications usually require special functions related to the physical phenomenon.

The major difference between the IoT and CPS is the presence or absence of cooperative operation among various services. Hence, IoT focus on cooperative application applied to various service domains. As a result, conventional protocols for CPSs are not enough to connect among different service domains, and many organizations and alliances such as IEEE (The Institute of Electrical and Electronics Engineers, Inc.), ITU-T (International

Faculty of Information Science, Aichi Institute of Technology, Toyota, Aichi 470-0392, Japan

a) naito@pluslab.org
Telecommunication Union Telecommunication Standardization Sector), 3GPP (Third Generation Partnership Project), ISO (International Organization for Standardization), IEC (International Electrotechnical Commission), etc. have been standardizing several protocols to realize horizontally-integrated applications [5].

Machine-to-machine (M2M) has a similar meaning of IoT [6]. However, the authors consider that M2M focuses on an automatic cooperation among machines comparing to IoT to realize a service. Since typical M2M devices do not have enough computation power due to hardware specifications, simplified protocols for resource constrained devices have been proposed. Industrie 4.0 [7] and Industrial Internet Consortium (IIC) [8] have considered standards for practical applications of $\mathrm{M} 2 \mathrm{M}$ in an industrial domain. They are trying to develop a new application platform for manufacturing floors such as a factory, manufacturing facility, etc. In near future, direct communication mechanisms among M2M devices are required to realize scalable and flexible service systems among different service domains [9]. As a result, further consideration should be required for practical M2M systems [10].

This paper presents a survey on the current state of the art of standards for IoT technologies. First, the author gives a brief introduction to related standards in Section 2. The survey in Section 3 gives an overview of research areas in IoT. In Section 4, the author also analyzes the issues in current IoT systems, and proposes an idea of the future platform of scalable IoT systems. Finally, we summarize our idea and take away some points in our conclusion in Section 5.

\section{Related Standard}

IoT requires various kinds of technologies. Especially, communication technologies are a fundamental framework to realize IoT services. This section introduces the main stream protocols and standards for IoT. Figure 1 shows the key wireless 


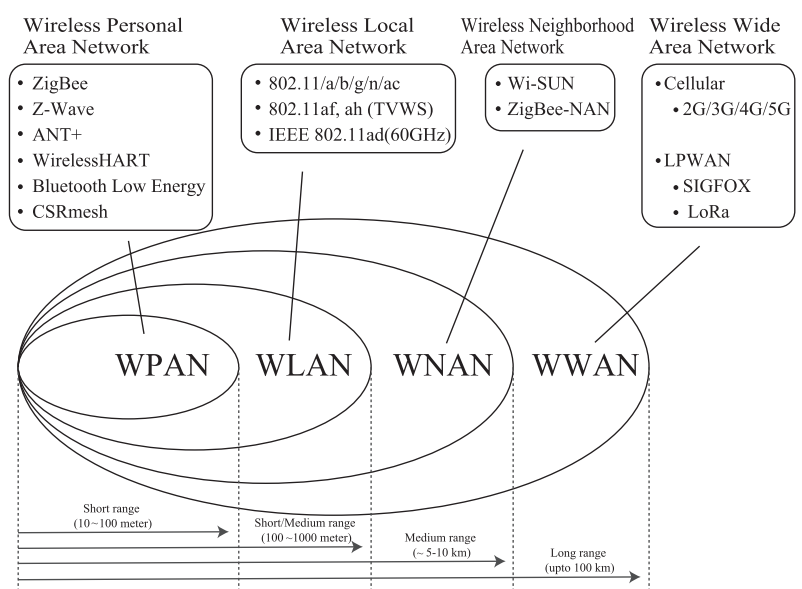

Fig. 1 Key wireless technologies for IoT.

technologies for IoT. The technologies are classified into WPAN (Wireless Personal Area Network), WLAN (Wireless Local Area Network), WNAN (Wireless Neighborhood Area Network), and WWAN (Wireless Wide Area Network). Detail technologies are described in the following subsection.

\subsection{WPAN/WNAN}

\section{IEEE 802.15.4}

IEEE 802.15.4 [11] is a standard for low-rate wireless personal area networks (LR-WPANs). Hence it focuses on low power consumption and low transmission rate $(250 \mathrm{Kbps})$ because devices are assumed to operate in a battery. It defines three types of nodes: PAN coordinator, full-function device (FFD) and reducedfunction devices (RFD). The nodes can construct either peer-topeer or star networks. Due to a limitation of devices, the maximum packet size is limited to 127 Bytes including a header and a payload.

IEEE 802.15.4g is an extended standard of IEEE 802.15.4 for low-rate wireless neighborhood area network, and facilitates very large scale process control applications such as Smart Utility Networks (SUN) with minimal infrastructure, with many fixed end-nodes. The target utility is metering of electricity, gas, etc. The IEEE $802.15 .4 \mathrm{~g}$ builds a smart WNAN and extends a data payload size (2,048 Bytes) comparing to the conventional IEEE 802.15.4. IEEE $802.15 .4 \mathrm{e}$ is a standard for MAC layer mechanisms to realize a low power intermittent operation. Typically, it is used with IEEE $802.15 .4 \mathrm{~g}$ because it does not define any physical layer.

IEEE $802.15 .4 \mathrm{k}$ is physical layer specifications for low energy critical infrastructure monitoring network. It provides a low transmission rate less than $10 \mathrm{Kbps}$ and a long-distance communication longer than $1 \mathrm{~km}$. As a result, IEEE 802.15.4 will be one of mainstream standards for IoT devices.

\section{Alliance}

There are some alliance to develop a product for IoT because almost all IEEE standards for wireless communication define fundamental specification and not enough to make a product with a mutual compatibility.

ZigBee [12], Wi-Sun [13], Thread [14], Wireless HART [15] are specifications based on IEEE 802.15.4. The latest version
3.0 of ZigBee provides a seamless interoperability among the widest range of smart devices. It also defines standard specifications at all levels of the network, especially the application level for practical services. Wi-Sun focuses on field area networks for applications such as advanced metering infrastructure, distribution automation, and home energy management. It also provides secure IPv6 communications over an IEEE 802.15.4g based wireless mesh network. Thread is an IPv6 based mesh network protocol, and provides the Thread networking stack on IEEE 802.15.4. Hence, each Thread end device can connect to the Internet through a native IP protocol. Wireless HART is a wireless standard expanding HART (Highway Addressable Remote Transducer) standard of a digital industrial automation protocol for process automation in factories. The benefit of Wireless HART is a backward compatibility to traditional HART instruments.

Z-Wave [16] is a wireless communications protocol on sub$\mathrm{GHz}$ band used for home automation, and provides reliable, lowlatency transmission of small data packets at data rates up to $100 \mathrm{Kbps}$. The physical and MAC layers of Z-Wave comply with recommendation ITU-T G.9959 [17]. It uses a source-routed mesh network architecture to deliver messages. Each Z-Wave network is identified by a Home ID, and each device is also identified by a Node ID. Z-Wave has two basic types of device: controllers for controlling other Z-wave devices and slaves that are controlled by other Z-Wave controllers. Z-Wave alliance also defines specific profiles for home automation like as ZigBee 3.0. Therefore, they guarantee an interoperability among devices of different vendors.

CSRmesh is a protocol running over Bluetooth Smart [18]. It provides message relaying over multiple Bluetooth Smart devices and enables consumer products such as smartphones, tablets, etc. employing Bluetooth Smart to interact directly with devices within the CSRmesh network.

IEEE 802.1 TSN (Time Sensitive Networking) [19] is an extension protocol of Ethernet AVB (IEEE802.1 Audio/Video Bridging). These extensions provide very low transmission latency and high availability. The aim of IEEE 802.1 TSN is realized deterministic communication that ensures data throughput, transmission delay, and real-time communication. The benefit of the standard is realizing real-time and time critical message deliveries on standardized Ethernet components.

\subsection{WLAN}

IoT devices use a communication function to connect to the Internet. The traditional standard is IEEE 802.3 [20], that is a well-known wired network standard called Ethernet. Some IoT devices employ Ethernet to connect to a network when the devices are fixed in a facility because power over Ethernet (PoE) can also provide electric power to devices. Recently, power consumption of IoT devices is rapidly reduced according to an advancement of semiconductor technology. As a result, recent IoT devices employ a wireless communication device to connect to the Internet. The following is the main stream standards to realize a local network.

IEEE 802.11 [21] is a well known standard for wireless LAN. 
Recent consumer WLAN devices support IEEE 802.11n/ac, which support Multi-Input Multi-Output (MIMO) technologies on $2.4 \mathrm{GHz}$ and $5 \mathrm{GHz}$ bands. Since the wireless bands for WLAN are limited and crowded now. New standards for different bands have been proposed.

IEEE 802.11ad is known as Wireless Gigabit (WiGig), that supports $7 \mathrm{Gbps}$ on $60 \mathrm{GHz}$ band. The purpose of IEEE 802.11 ad is to provide a high throughput performance in a limited area because a $60 \mathrm{GHz}$ signal attenuates rapidly according to a distance.

IEEE 802.11af employs a similar Orthogonal Frequency Division Multiplexing (OFDM) technology on TeleVision White Space frequency spectrum (TVWS) that is sub-GHz bands. A bandwidth of the OFDM signal in IEEE 802.11 af is $6 \mathrm{MHz}$ or $7 \mathrm{MHz}$ that is a same bandwidth of a TV broadcasting signal. Since TV towers also use TVWS band, Internet Engineering Task Force (IETF) offers a standard for channel sensing methods. Typically, IoT devices should check a TVWS database or sense a signal from TV towers. IEEE 802.11af will be a main stream standard to provide high throughput performance on TVWS.

IEEE 802.11 ah is a similar standard of IEEE 802.11 af because both standards use a TVWS band. However, the target of both standards is especially different. The IEEE 802.11ah focuses on a long-distance communication with low power consumption. Hence, it also supports $1 \mathrm{MHz}$ and $2 \mathrm{MHz}$ bands to extend a communication distance. Therefore, IEEE 802.11 ah should be a core stream standard in IEEE 802.11 series for IoT devices because typical IoT devices require a long communication distance instead of high throughput performance.

\subsection{WWAN}

Typical IoT services are employing cloud services to provide functions to end-IoT devices. Therefore, communication technologies for wide area networks are also important to realize practical IoT services. The following is the main stream standards for wide area networks.

\section{GPP}

3GPP (Third Generation Partnership Project) [22] has developed standards for cellular network systems. The traditional target of the standards is to realize a high-speed communication. On the contrary, recent trends for $4 \mathrm{G}$ and $5 \mathrm{G}$ cellular systems have two categories: high-speed communication and low-speed communication with low power consumption. For examples, LTEAdvanced Release 13 focuses on a new specification for IoT devices, and defines new terminal categories: category M1 that supports a narrow band communication and NB(NarrowBnad)-IoT that limits a bandwidth less than $180 \mathrm{KHz}$. Additionally, it also updates the specification of PSM (Power Saving Mode), and defines an extended DRX (Discontinuous Reception) to extend an intermittent reception interval of a paging mechanism to reduce the consumed power.

\section{GSMA/eSIM}

The GSMA's eSIM (Embedded SIM) [23] specification provides a standard mechanism for remote provisioning and management of machine to machine (M2M) connections. Traditional cellular devices require a physical traditional SIM card to connect an operator network. Therefore, products prepare a special card slot for a SIM card and customers should install a SIM card into the products. GSMA/eSIM assumes Embedded Universal Integrated Circuit Card (eUICC) that is a new embedded SIM function. The eUICC identification information (eICCiD) has an operational profile working as a traditional provisioning profile and provisioning profile for downloading an operational profile. Therefore, it allows the "over the air" provisioning of an initial operator subscription, and the subsequent change of subscription from one operator to another. An eSIM selects a profile from installed profiles according to a direction from a subscription manager operated by a mobile network operator.

\section{LPWAN}

LPWAN (Low-Power, Wide-Area Networks) is a new category of a wireless communication technology that supports longdistance communication, low data rate and low power consumption. This paper introduces SIGFOX [24] and LoRa [25] as concrete examples.

SIGFOX employs an ultra-narrow band (UNB) technology and builds a cellular style system to serve communication service. Therefore, it is mobile network operators adopting their technology for IoT deployments. It also opens its technologies of endpoints for any vendors to develop their own products. Endpoints use bidirectional communication in the specification of SIGFOX. However, appropriate density of base stations is required to provide a high quality bidirectional communication service.

LoRa is also a LPWAN specification intended for wireless battery operated things. A topology of LoRa is a star topology in which base stations are a transparent bridge relaying messages between endpoints and a server. The data rates of LoRa are from $0.3 \mathrm{Kbps}$ to $50 \mathrm{Kbps}$. Since the radio devices for an endpoint and a basestation are almost same specification, a basestation of LoRa is not so expensive comparing to that of SIGFOX. Unlike SIGFOX, LoRa provides a symmetric link for endpoints.

\subsection{Constrained Protocols}

IoT typically assumes constrained nodes and constrained networks. Therefore, historical full stack protocols are not appropriate to deploy on constrained nodes. As a result, constrained protocols have been considered for an IP network and application layers.

\section{LowPAN}

6LowPAN (IPv6 over Low power Wireless Personal Area Networks) [26], [27] has been designed for low-power devices with limited processing capabilities. The 6LoWPAN group has defined encapsulation and header compression mechanisms. Hence, IPv6 packets can be sent and received over IEEE 802.15.4 based networks. 6LowPAN provides some functions: adapting the packet sizes between a traditional IP network and an IEEE 802.15.4 network, and address resolution between IPv6 addresses and IEEE 64 bit extended addresses on IEEE 802.15.4, adaptation layer for interoperability and packet formats between IPv6 domain and IEEE 802.15.4 domain. Figure 2 shows the overview of fragmentation in 6LowPAN. Since the maximum message size is limited up to 128 bytes in IEEE 802.15.4, the fragmentation process is optimized to covey an IPv6 payload effectively. 6LoWPAN uses a dispatch field that is the first part of a packet to recog- 


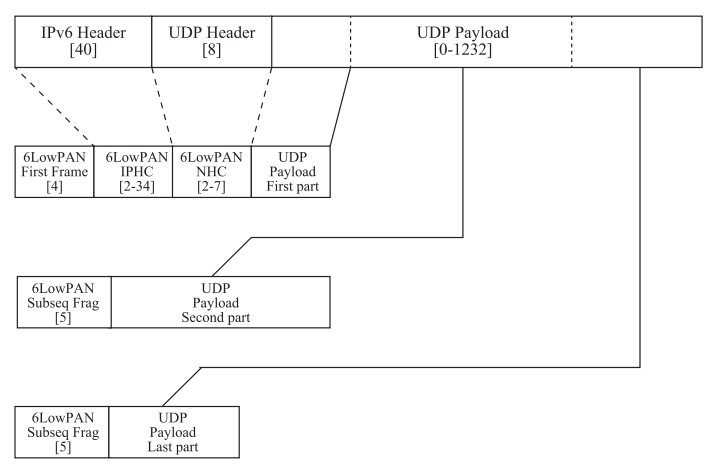

Fig. 2 Fragmentation in 6LowPAN.

nize a type of the packet. It defines two types of dispatches: first fragment and subsequent fragments for carrying an IP datagram. The first fragment carries a compressed IPv6 header information, a transport layer header and a first part of a payload. On the contrary, the subsequent fragments carry only a part of the payload of an IPv6 datagram because the compressed IPv6 header should be an overhead in the limited payload size of IEEE 802.15.4.

\section{CoAP}

CoAP (Constrained Application Protocol) [28] is a simple application layer protocol for very simple electronics devices. it is designed to translate to Hypertext Transfer Protocol (HTTP) easily for simplified integration with web systems, and provides a request/response interaction model between application endpoints Hence proxying between CoAP and HTTP can easily translate messages through an intermediary. It uses a short fixed-length binary header ( 4 bytes). Since it defines two messages types of requests and responses, it uses two simple message types: requests and responses. The header format is shared by these message types. Each message contains a message ID used to detect duplicates. The message procedures in CoAP are carried with either a method code or a response code, respectively. it is bound to User Datagram Protocol (UDP) and optionally to Datagram Transport Layer Security (DTLS) to provide a high level of communication security.

\section{MQTT}

MQ Telemetry Transport (MQTT) [29] is a lightweight publish/subscribe messaging protocol. Multiple clients connect to a broker and subscribe to topics, and also connect to the broker and publish messages to topics. Since topics are treated as a hierarchy, clients can handle all topics in the same way as a filesystem. MQTT defines three levels of Quality of Service (QoS): QoS 0 for delivering a message once with no confirmation, QoS 1 for delivering a message at least once with confirmation required and QoS 2 for delivering a message exactly once by using a four step handshake. Therefore, publishers and clients can control the level of delivery QoS according to their service model. Since the MQTT protocol requires an underlying transport that provides an ordered and reliable communication, TCP is used for MQTT. Additionally, TLS is used to realize a secure function on the MQTT protocol.

\subsection{IoT Platform}

Messages in IoT systems travel from an end device to an appli- cation system through PAN, WAN and a platform layer. Recently, several platform layer standards have been discussed in some organizations.

\section{OneM2M}

OneM2M [30] provides a common M2M service layer that can be readily embedded within various hardware and software. It also defines use cases and requirements for a common set of service, protocols and APIs, security and privacy mechanisms, interoperability, identification and naming of devices and applications, information models and data management, and management aspects. The benefit of OneM2M is to consider horizontal service domains in IoT to reuse information and to create a new value from the reused information.

\section{IEEE 2413}

IEEE 2413 [31] defines an architectural framework for the IoT. The framework includes descriptions of IoT domains, definitions of IoT domain abstractions, and identification of commonalities between different IoT domains. It also provides some reference architectures to build a reference model according to a practical service application. Especially it focuses on cross-domain interaction, system interoperability and functional compatibility in the IoT.

\section{WoT}

Web of Things (WoT) [32] is a new term to handle real-world objects to be part of the World Wide Web. It provides a simplified application layer to create IoT products. Since WoT uses HTML5/JavaScript as a developing language, developed code should work on various kinds of hardwares and OSs. Additionally, WoT defines standards to obtain information through a Web API. As a result, Web applications on every IoT device can connect each other through Web APIs.

\section{Research Area in IoT}

IoT services are built up by a wide range of research domains. This section introduces research domains for IoT briefly.

\subsection{Application in IoT}

Original idea of IoT application relies on sensor network applications. Hence, early researches focuses on developing a smart monitoring system with wireless sensor and actuator networks [33]. Since a wireless band is limited and IP based networks are important in practical services, rate control mechanisms and IP based sensor networks have been considered [34], [35], [36], [37], [38], [39], [40]. Recently, IoT service expands to cover health care applications to recognize human activities [41], [42]. Additionally, Vehicular Adhoc NETworks (VANETs) also focus on IoT from the view point of M2M communication [43], [44] because connected vehicles are essentially a network of machines communicating each other.

\subsection{Cloud Services in IoT}

Cloud services are a core function to provide valuable services in IoT. Therefore, cloud based IoT platforms have been developed for a specific service because standardization on horizontal service domains are still under working [45], [46], [47]. Additionally, some researchers have tried to port an application logic 
from firmware on a device to a cloud service because the moving of an application logic means a flexible software management on embed devices [48].

According to the technical advantages of IoT devices, fog and edge computing are a new topic of interest to researchers [49]. Since network capacity for IoT is limited in real wireless systems [50], reducing the amount of information at IoT devices is effective to realize flexible and scalable IoT systems [51]. Hence, some frameworks for edge computing have been proposed to realize a distributed programming model [52].

\subsection{Protocols}

IoT services require wireless networks to easily deploy IoT devices. As a result, routing protocols are an important function to realize practical wireless networks. ADOV [53] and DSR [54] are the well-known routing protocols for ad-hoc networks [55], and a geographic routing is also suitable for unstable networks such as vehicular networks [56].

6LowPAN in IoT practically assume RPL (IPv6 Routing Protocol for Low power and Lossy Networks) as a routing protocol [57], [58], [59]. Hence, an implementation has been developed on real devices [60], [61], [62], [63], and evaluation software have also proposed [64], [65], [66]. Additionally, some researchers have evaluated performance on RPL under mobility to apply RPL to VANETs [67]. IoT services also depend on upper layer protocols such as an application layer [68], [69]. Some researches have developed CoAP on real devices [70], [71]

\subsection{Security}

Security and privacy are becoming a major concern of IoT systems because IoT devices may be installed in private areas and collected information may be valuable [72], [73] Some practical CoAP applications employ DTLS to realize a high security level of communication [74], [75]. Some researchers proposed a special virtual network for IoT service to protect information [76]. Additionally, lightweight ciphers have also proposed for the IoT because IoT devices may be resource constrained devices [77].

\section{Scalable and Interoperable IoT}

Recent IoT services have been developed on a vertical system model, where every layer has been designed by a company or an organization. As a result, a recent trend of standardization considers a horizontal system model to realize scalable and interoperable operations in IoT services. As this paper has introduced, various standard protocols have been proposed to establish a cooperative mechanism among horizontal service domains. These protocols usually focus on a standardization in an application layer. Therefore, they assume that the inter-accessibility among end-nodes should be guaranteed. On the contrary, practical IP networks have some issues for inter-accessibility due to a difference of IP protocol version and firewalls. This section proposes a new layer design for IoT service to realize inter-operation among end-nodes in different networks.

\subsection{System Model}

A current layer model for IoT assumes that IP networks are

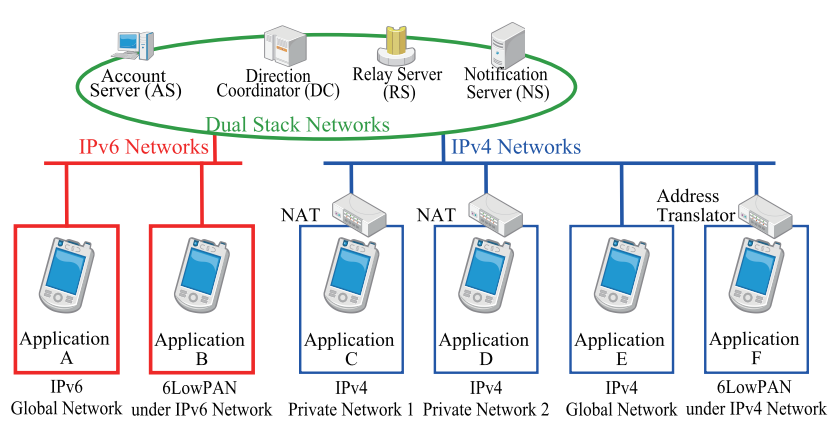

Fig. 3 System model of NTMobile.

transparent, that means every end-nodes can access each other. This assumption should be reasonable when an IoT service is operated in a closed IP network such as smart metering systems. The author considers that IoT should consider an open IP network in the near future because constructing a closed IP network is difficult when different vendors provide IoT devices and these devices cooperate each other. As a result, the author considers that a new layer model should have a middleware layer between the IP layer and the transport layer to realize a transparent connectivity among end-nodes.

The proposed model has a new layer to realize a transparent connectivity over IP networks because we should solve accessibility issues due to the different protocol versions, NAT traversal, and mobility. The proposed idea is to employ an IP mobility protocol or Information-Centric Networking (ICN). Since ICN provides some features such as topology independent name based routing, mobility support and packet level security [78], some designs have been proposed recently [79], [80], [81]. As the author has developed an IP mobility mechanism, this paper focuses on an integration of IP mobility protocol and IoT services.

\subsection{IP Mobility Based Overlay Network}

The author has developed a new IP mobility technology called NTMobile (Network Traversal with Mobility) [82], [83]. NTMobile can provide useful features such as an IP mobility and accessibility in both IPv4 and IPv6 networks. Since end-nodes use virtual IP addresses that are independent addresses from physical IP addresses, end-nodes can continuously communicate with each other when they switch an access network.

Figure 3 shows the system model of NTMobile. NTMobile consists of some management servers:account server (AS), direction coordinator (DC), notification server (NS), relay server (RS) and many end-nodes called NTMobile clients. AS serves an authentication service for NTMobile clients when they connect to the NTMobile network. DC manages communication among NTMobile clients according to their mobility status. RS relays communication among NTMobile clients when a direct connection is not available due to the different protocol IP version or a specification of routers. NS manages incoming connections to NTMobile clients.

\subsection{Naming Space}

The naming space in NTMobile employs DNS (Domain Name System) mechanisms because typical upper layer applications use DNS mechanisms to solve host names. Therefore, NTMobile 


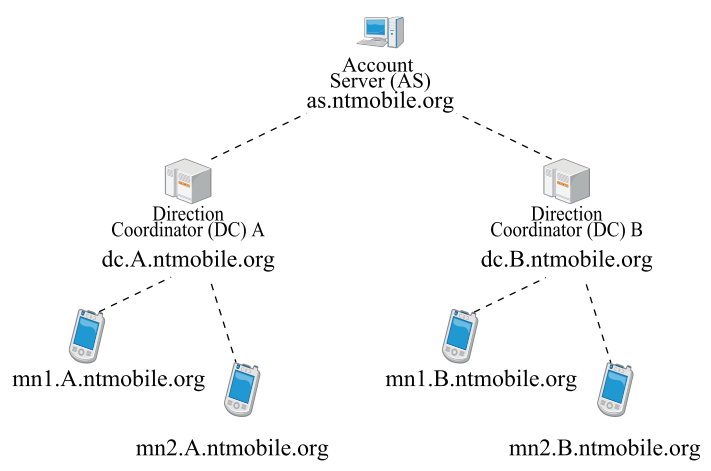

Fig. 4 Naming space of NTMobile.

\begin{tabular}{|c|c|c|c|}
\hline Application & & & Application \\
\hline CoAP / MQTT & & & CoAP / MQTT \\
\hline $\begin{array}{c}\text { UDP / TCP } \\
\text { Virtual IPv6 address }\end{array}$ & & & $\frac{\text { UDP / TCP }}{\text { Virtual IPv6 address }}$ \\
\hline NTMobile & Addres & anslator & NTMobile \\
\hline 6LowPAN & 6LowPAN & IPv4 / IPv6 & IPv4 / IPv6 \\
\hline $\begin{array}{l}\text { Network } \\
\text { Interface }\end{array}$ & $\begin{array}{l}\text { Network } \\
\text { Interface }\end{array}$ & $\begin{array}{l}\text { Network } \\
\text { Interface }\end{array}$ & $\begin{array}{l}\text { Network } \\
\text { Interface }\end{array}$ \\
\hline
\end{tabular}

Fig. 5 Interoperability to 6LowPAN networks with NTMobile.

specification requests DC to implement a DNS server function to manage its domain. Since DNS is originally a distributed database system, the naming space of NTMobile has also a similar distributed database manner. Hence, NTMobile clients can communicate with each other even if different DC manages each NTMobile client because each DC can easily find each other by using DNS mechanisms.

Figure 4 shows the overview of naming space in NTMobile. AS manages the top domain of NTMobile naming space because AS signs a digital certificate of each server. Therefore, each DC has own subdomain space. Since each DC has own domain name, each NTMobile client has also Fully Qualified Domain Name (FQDN) with DC's subdomain. As a result, every NTMobile client can recognize each other by using FQDN in the NTMobile network.

\subsection{Interoperability to 6LowPAN Networks}

The basic idea of IP mobility in NTMobile is employing a virtual IP address to make a connection. Therefore, NTMobile can use both IPv4 and IPv6 for virtual IP addresses. As this paper presented, recent trends in IoT services assumes full IP connectivity to end-devices, that means IPv6 addresses are used with the 6LowPAN mechanism. Therefore, NTMobile should use IPv6 virtual addresses to realize interoperability with IoT services.

Figure 5 shows the protocol stacks between a 6LowPAN network and a traditional IP network. The function of NTMobile is inserted between the IP layer and the transport layer. Therefore, NTMobile provides a virtual IPv6 address to the transport layer, and the transport layer can manage a connection by the virtual IPv6 address. NTMobile can capsulate a datagram with the virtual IP address into new datagram with an IPv4 or an IPv6 address. Hence, IoT devices can communicate with each other through a 6LowPAN network.

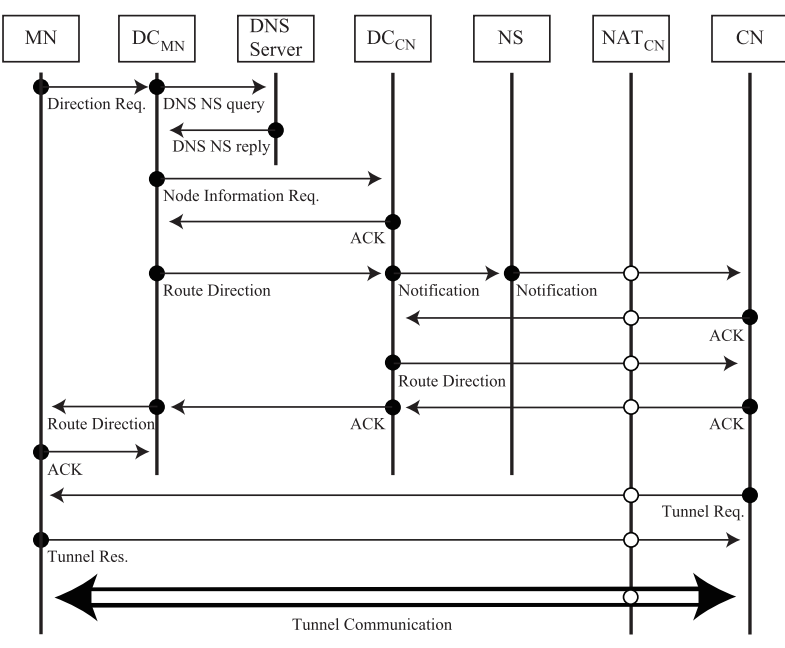

Fig. 6 Example signaling process (Global to Private).

\subsection{Security of NTMobile}

NTMobile provides secure communication based on Advanced Encryption Standard (AES) encryption between NTMobile clients. Since the encryption key is transferred directly between NTMobile clients, privacy of NTMobile communication is guaranteed. As exchange mechanisms of the encryption key, NTMobile has two methods with a temporary key or a digital certificate. In the temporary key method, DC distributes a temporary key for exchanging a communication key to both NTMobile clients when both clients start communication. NTMobile guarantees secure communication among whole nodes including AS, DC, RS, NS, and NTMobile clients with a different shared encryption key. Therefore, the communication key is secretly shared by both NTMobile clients. In the digital certificate method, each NTMobile client has own digital certificate signed by their AS. They also verify a certificate each other, and exchange a communication key.

\subsection{Example Signaling Process of NTMobile}

NTMobile provides some signaling processes to realize a tunnel communication between NTMobile clients according to a network condition. Figure 6 shows the example of the signaling process where one NTMobile client has a global IPv4 address and the other NTMobile client has a private IPv4 address. This figure defines MN (Mobile Node) and CN (Correspondent Node) as two NTMobile clients. The initiation process starts at MN side, and $\mathrm{MN}$ transmits the direction request message to $\mathrm{DC}_{\mathrm{MN}}$. $\mathrm{DC}_{\mathrm{MN}}$ tries to find $\mathrm{DC}_{\mathrm{CN}}$ by checking NS record of DNS because each DC is a DNS server for its own domain. Then, it also transmits the node information request message to $\mathrm{DC}_{\mathrm{CN}}$ to obtain the information of CN. Since it can select a signaling process according to the network condition, it transmits the route direction message that indicates the signaling process. In this example, the route direction message indicates $\mathrm{CN}$ to transmit a tunnel request message because $\mathrm{CN}$ exists behind the NAT router. NS informs CN that the direction message arrives at $\mathrm{DC}_{\mathrm{CN}}$. Then, $\mathrm{CN}$ obtains the direction message from $\mathrm{DC}_{\mathrm{CN}}$. $\mathrm{DC}_{\mathrm{MN}}$ also transmits the direction message when it receives the acknowledgement from $\mathrm{CN}$. In this example, $\mathrm{CN}$ transmits the tunnel request message and $\mathrm{MN}$ 
replies the tunnel reply message to establish the tunnel communication. Detail signaling procedures of NTMobile can be found in the reference papers [82], [83].

\section{Conclusion}

This paper has presented a survey on recent technologies and standards for IoT. Since early IoT systems have been developed in a vertical service model, an interoperability among different service domains is a big issue in recent day. As a result, the latest trend of IoT technologies is to consider horizontal service domains to realize an interoperability, and many alliances for IoT has proposed their standardized protocols. However, current IoT does not make an inter-operation among IoT devices in different networks. This paper also proposes an idea of the future platform of scalable IoT systems. The proposed idea employs IP mobility technologies to realize inter-operability among devices in different networks. Additionally, the proposed idea is excellent with 6LowPAN mechanisms that are used in IoT networks. Therefore, the author believes that the proposed idea will be one of IoT platforms to realize a remotely-connected operation among IoT devices.

Acknowledgments This work is supported in part by Grantin-Aid for Scientific Research (B) (15H02697) and Grant-in-Aid for Scientific Research (C) (26330103), Japan Society for the Promotion of Science (JSPS) and the Integration research for agriculture and interdisciplinary fields, Ministry of Agriculture, Forestry and Fisheries, Japan.

\section{References}

[1] Sheng, Z., Yang, S., Yu, Y., Vasilakos, A.V., Mccann, J.A. and Leung, K.K.: A survey on the ietf protocol suite for the internet of things: Standards, challenges, and opportunities, IEEE Wireless Communications, Vol.20, No.6, pp.91-98 (Dec. 2013).

[2] Khaitan, S.K. and McCalley, J.D.: Design techniques and applications of cyberphysical systems: A survey, IEEE Systems Journal, Vol.9, No.2, pp.350-365 (June 2015).

[3] Hahm, O., Baccelli, E., Petersen, H. and Tsiftes, N.: Operating systems for low-end devices in the internet of things: A survey, IEEE Internet of Things Journal, Vol.3, No.5, pp.720-734 (Oct. 2016).

[4] Montenegro, G., Kushalnagar, N., Hui, J. and Culler, D.: Transmission of ipv6 packets over ieee 802.15.4 networks (Sep. 2007).

[5] Al-Fuqaha, A., Guizani, M., Mohammadi, M., Aledhari, M. and Ayyash, M.: Internet of things: A survey on enabling technologies, protocols, and applications, IEEE Communications Surveys $\mathcal{E}$ Tutorials, Vol.17, No.4, pp.2347-2376 (2015).

[6] Kim, J., Lee, J., Kim, J. and Yun, J.: M2m service platforms: Survey, issues, and enabling technologies, IEEE Communications Surveys $\mathcal{E}$ Tutorials, Vol.16, No.1, pp.61-76 (2014).

[7] Industrie 4.0, available from 〈http://www.plattform-i40.de〉.

[8] Industrial Internet Consortium, available from 〈https://www.iiconsortium.org $\rangle$.

[9] Sabella, D., Vaillant, A., Kuure, P., Rauschenbach, U. and Giust, F.: Mobile-edge computing architecture: The role of mec in the internet of things, IEEE Consumer Electronics Magazine, Vol.5, No.4, pp.8491 (Sep. 2016).

[10] Kafle, V.P., Fukushima, Y. and Harai, H.: Internet of things standardization in itu and prospective networking technologies, IEEE Communications Magazine, Vol.54, No.9, pp.43-49 (Sep. 2016).

[11] IEEE. Ieee 802.15: Wireless personal area networks (pans), available from 〈https://standards.ieee.org/about/get/802/802.15.html〉.

[12] ZigBee Alliance: Zigbee 3.0, available from $\langle$ http://www.zigbee.org/zigbee-for-developers/zigbee3-0/〉.

[13] Wi-SUN Alliance, available from 〈http://www.wi-sun.org $\rangle$.

[14] Thread, available from $\langle$ http://threadgroup.org $\rangle$.

[15] HART Communication Foundation: Wireless hart, available from $\langle$ http://en.hartcomm.org .

[16] Z-Wave Alliance, available from 〈http://z-wavealliance.org $\rangle$.
[17] ITU-T. G.9959: Short range narrow-band digital radiocommunication transceivers - phy, mac, sar and llc layer specifications, available from $\langle$ https://www.itu.int/rec/T-REC-G.9959〉.

[18] Bluetooth SIG: Bluetooth low energy, available from 〈https://www.bluetooth.com/what-is-bluetooth-technology/ bluetooth-technology-basics/low-energy .

[19] IEEE: Ieee 802.1 time-sensitive networking task group, available from 〈http://www.ieee802.org/1/pages/tsn.html〉.

[20] IEEE: Ieee 802.3 ethernet working group, available from 〈http://www.ieee802.org/3/〉.

[21] IEEE: Ieee $802.11 \mathrm{tm}$ wireless local area networks, available from 〈http://www.ieee802.org/11/〉.

[22] 3rd Generation Partnership Project, available from 〈http://www.3gpp.org .

[23] GSMA: Remote sim provisioning for machine to machine, available from 〈http://www.gsma.com/connectedliving/embedded-sim/ $\rangle$.

[24] SIGFOX, available from 〈https://www.sigfox.com $\rangle$.

[25] LoRa Alliance, available from $\langle$ https://www.lora-alliance.org $\rangle$.

[26] Geoff Mulligan: The 6lowpan architecture, Proc. 4th Workshop on Embedded Networked Sensors, pp.78-82, New York, NY, USA, ACM (2007).

[27] Hui, J., Culler, D. and Chakrabarti, S.: 6lowpan: Incorporating ieee 802.15. 4 into the ip architecture (2009).

[28] Constrained Application Protocol, available from $\langle$ http://coap.technology .

[29] International Business Machines Corporation (IBM), Mqtt v3.1 protocol specification.

[30] OneM2M, available from 〈http://www.onem2m.org .

[31] IEEE: P2413 - standard for an architectural framework for the internet of things (iot), available from /https://standards.ieee.org/develop/ project/2413.html .

[32] Web of Things, available from 〈http://webofthings.org $\rangle$.

[33] Bressan, N., Bazzaco, L., Bui, N., Casari, P., Vangelista, L. and Zorzi, M.: The deployment of a smart monitoring system using wireless sensor and actuator networks, 2010 First IEEE International Conference on Smart Grid Communications (SmartGridComm), pp.49-54 (Oct. 2010).

[34] Sridharan, A. and Krishnamachari, B.: Explicit and precise rate control for wireless sensor networks, Proc. 7th ACM Conference on Embedded Networked Sensor Systems, pp.29-42, ACM (2009).

[35] Yazar, D. and Dunkels, A.: Efficient application integration in ip-based sensor networks, Proc. First ACM Workshop on Embedded Sensing Systems for Energy-Efficiency in Buildings, pp.43-48, ACM (2009).

[36] Duquennoy, S., Wirström, N., Tsiftes, N. and Dunkels, A.: Leveraging ip for sensor network deployment, Proc. Workshop on Extending the Internet to Low Power and Lossy Networks (IP+SN 2011), Chicago, IL, USA, Vol.11 (2011).

[37] Ishaq, I., Hoebeke, J., Moerman, I. and Demeester, P.: Internet of things virtual networks: Bringing network virtualization to resourceconstrained devices, 2012 IEEE International Conference on Green Computing and Communications (GreenCom), pp.293-300 (Nov. 2012).

[38] Altmann, V., Skodzik, J., Golatowski, F. and Timmermann, D.: Investigation of the use of embedded web services in smart metering applications, IECON 2012 - 38th Annual Conference on IEEE Industrial Electronics Society, pp.6172-6177 (Oct. 2012).

[39] Yang, S., Yang, X., McCann, J.A., Zhang, T., Liu, G. and Liu, Z.: Distributed networking in autonomic solar powered wireless sensor networks, IEEE Journal on Selected Areas in Communications, Vol.31, No.12, pp.750-761 (Dec. 2013).

[40] Lee, I.-G. and Kim, M.: Interference-aware self-optimizing wi-fi for high efficiency internet of things in dense networks, Computer Communications, pp.60-74 (Mar. 2016).

[41] Woznowski, P., Kaleshi, D., Oikonomou, G. and Craddock, I.: Classification and suitability of sensing technologies for activity recognition, Computer Communications, pp.34-50 (Mar. 2016).

[42] Mano, L.Y., Faiçal, B.S., Nakamura, L.H.V., Gomes, P.H., Libralon, G.L., Meneguete, R.I., Filho, P.R.G., Giancristofaro, G.T., Pessin, G., Krishnamachari, B. et al.: Exploiting iot technologies for enhancing health smart homes through patient identification and emotion recognition, Computer Communications, pp.178-190 (Mar. 2016).

[43] Kumar, Y., Kumar, P. and Kadian, A.: A survey on routing mechanism and techniques in vehicle to vehicle communication (vanet), International Journal of Computer Science $\mathcal{E}$ Engineering Survey, Vol.2, No.1 (2011).

[44] Booysen, M.J., Gilmore, J.S., Zeadally, S. and Van Rooyen, G.-J. Machine-to-machine $(\mathrm{m} 2 \mathrm{~m})$ communications in vehicular networks, Ksii Trans. Internet and Information Systems (2012).

[45] Lea, R. and Blackstock, M.: City hub: A cloud-based iot platform for smart cities, 2014 IEEE 6th International Conference on Cloud Computing Technology and Science (CloudCom), pp.799-804 (Dec. 
2014).

[46] Sivieri, A., Mottolaa, L. and Cugola, G.: Building internet of things software with eliot, Computer Communications, pp.141-153 (Feb. 2016).

[47] Pfisterer, D., Romer, K., Bimschas, D., Kleine, O., Mietz, R., Truong, C., Hasemann, H., Kröller, A., Pagel, M., Hauswirth, M., Karnstedt, M., Leggieri, M., Passant, A. and Richardson, R.: Spitfire: Toward a semantic web of things, IEEE Communications Magazine, Vol.49, No.11, pp.40-48 (Nov. 2011).

[48] Kovatsch, M., Mayer, S. and Ostermaier, B.: Moving application logic from the firmware to the cloud: Towards the thin server architecture for the internet of things, Proc. 2012 6th International Conference on Innovative Mobile and Internet Services in Ubiquitous Computing, pp.751-756, IEEE Computer Society (2012).

[49] Bonomi, F., Milito, R., Zhu, J. and Addepalli, S.: Fog computing and its role in the internet of things, Proc. 1st Edition of the MCC Workshop on Mobile Cloud Computing, pp.13-16, ACM (2012).

[50] Maia, A.M., Vieirab, D., de Castroa, M.F. and Ghamri-Doudane, Y.: A fair qos-aware dynamic lte scheduler for machine-to-machine communication, Computer Communications, pp.1-12 (Sep. 2016).

[51] Borgia, E., Bruno, R., Conti, M., Mascitti, D. and Passarella, A.: Mobile edge clouds for information-centric iot services, 2016 IEEE Symposium on Computers and Communication (ISCC), pp.422-428 (June 2016).

[52] Giang, N.K., Blackstock, M., Lea, R. and Leung, V.C.M.: Developing iot applications in the fog: A distributed dataflow approach, 2015 th International Conference on the Internet of Things (IOT), pp.155-162 (Oct. 2015).

[53] Perkins, C., Ratliff, S. and Dowdell, J.: Dynamic manet on-demand (aodvv2) routing, draft-ietf-manet-dymo-26 (Feb. 2013).

[54] Johnson, D.: The dynamic source routing protocol (dsr) for mobile ad hoc networks for ipv4, Request for Comments: 4728 (Feb. 2007).

[55] Perkins, C.E., Royer, E.M., Das, S.R. and Marina, M.K.: Performance comparison of two on-demand routing protocols for ad hoc networks, IEEE Personal Communications, Vol.8, No.1, pp.16-28 (Feb. 2001).

[56] Jerbi, M., Senouci, S.M., Rasheed, T. and Ghamri-Doudane, Y.: Towards efficient geographic routing in urban vehicular networks, IEEE Transactions on Vehicular Technology, Vol.58, No.9, pp.5048-5059 (Nov. 2009).

[57] Clausen, T., Herberg, U. and Philipp, M.: A critical evaluation of the ipv6 routing protocol for low power and lossy networks (rpl), 2011 IEEE 7th International Conference on Wireless and Mobile Computing, Networking and Communications (WiMob), pp.365-372 (Oct. 2011).

[58] Pavković, B., Theoleyre, F. and Duda, A.: Multipath opportunistic rpl routing over ieee 802.15.4, Proc. 14th ACM International Conference on Modeling, Analysis and Simulation of Wireless and Mobile Systems, MSWiM'11, pp.179-186, ACM (2011).

[59] Ko, J.G., Dawson-Haggerty, S., Gnawali, O., Culler, D. and Terzis, A.: Evaluating the performance of rpl and 6lowpan in tinyos, Workshop on Extending the Internet to Low Power and Lossy Networks $(I P+S N)$, Vol.80, pp.85-90 (2011).

[60] Cody-Kenny, B., Guerin, D., Ennis, D., Carbajo, R.S., Huggard, M. and Mc Goldrick, C.: Performance evaluation of the 6lowpan protocol on micaz and telosb motes, Proc. 4th ACM Workshop on Performance Monitoring and Measurement of Heterogeneous Wireless and Wired Networks, pp.25-30, ACM (2009).

[61] Dawans, S., Duquennoy, S. and Bonaventure, O.: On link estimation in dense rpl deployments, 2012 IEEE 37th Conference on Local Computer Networks Workshops (LCN Workshops), pp.952-955 (Oct. 2012).

[62] Baccelli, E., Philipp, M. and Goyal, M.: The p2p-rpl routing protocol for ipv6 sensor networks: Testbed experiments, 2011 19th International Conference on Software, Telecommunications and Computer Networks (SoftCOM), pp.1-6 (Sep. 2011).

[63] Oikonomou, G. and Phillips, I.: Stateless multicast forwarding with $\mathrm{rpl}$ in 6lowpan sensor networks, 2012 IEEE International Conference on Pervasive Computing and Communications Workshops (PERCOM Workshops), pp.272-277 (Mar. 2012).

[64] Hammerseth, S.: Implementing rpl in a mobile and fixed wireless sensor network with omnet++ (Nov. 2011).

[65] Saad, L.B., Chauvenet, C. and Tourancheau, B.: Simulation of the rpl routing protocol for ipv6 sensor networks: Two cases studies, International Conference on Sensor Technologies and Applications SENSORCOMM 2011 (Sep. 2011).

[66] Herberg, U. and Clausen, T.: A comparative performance study of the routing protocols load and rpl with bi-directional traffic in lowpower and lossy networks (lln), Proc. 8th ACM Symposium on Performance Evaluation of Wireless Ad Hoc, Sensor, and Ubiquitous Networks, pp.73-80, ACM (2011).

[67] Lee, K.C., Sudhaakar, R., Ning, J., Dai, L., Addepalli, S., Vasseur, J.P. and Gerla3, M.: A comprehensive evaluation of rpl under mobility, Vehicular Technology (2012)

[68] Bormann, C., Castellani, A.P. and Shelby, Z.: Coap: An application protocol for billions of tiny, IEEE Internet Computing, pp.62-67 (Mar. 2012).

[69] Chen, Y., Chanet, J.P. and Hou, K.M.: Rpl routing protocol a case study: Precision agriculture, 1st China-France Workshop on Future Computing Technology (CF-WoFUCT 2012) (Feb. 2012).

[70] Kuladinithi, K., Bergmann, O., Pötsch, T., Becker, M. and Görg, C.: Implementation of coap and its application in transport logistics, Proc. $I P+S N$, Chicago, IL, USA (Jan. 2011).

[71] Becker, M., Pötsch, T., Kuladinithi, K. and Goerg, C.: Deployment of coap in transport logistics, Proc. 36th IEEE Conference on Local Computer Networks (LCN), Bonn Germany 2011 (2011).

[72] Kavitha, T. and Sridharan, D.: Security vulnerabilities in wireless sensor networks: A survey, Journal of information Assurance and Security, Vol.5, No.1, pp.31-44 (2010).

[73] Cui, A. and Stolfo, S.J.: A quantitative analysis of the insecurity of embedded network devices: Results of a wide-area scan, Proc. 26th Annual Computer Security Applications Conference, pp.97-106, $\operatorname{ACM}(2010)$.

[74] Brachmann, M., Garcia-Morchon, O. and Kirsche, M.: Security for practical coap applications: Issues and solution approaches, Proc. 10th GI/ITG KuVS Fachgespraech Sensornetze (FGSN11), Paderborn, Germany, pp.15-16 (2011).

[75] Raza, S., Trabalza, D. and Voigt, T.: 6lowpan compressed dtls for coap, 2012 IEEE 8th International Conference on Distributed Computing in Sensor Systems, pp.287-289 (May 2012).

[76] Ishaq, I., Hoebeke, J., Rossey, J., De Poorter, E., Moerman, I. and Demeester, P.: Facilitating sensor deployment, discovery and resource access using embedded web services, 2012 6th International Conference on Innovative Mobile and Internet Services in Ubiquitous Computing (IMIS), pp.717-724 (July 2012).

[77] Dinu, D., Le Corre, Y., Khovratovich, D., Perrin, L., Großschädl, J. and Biryukov, A.: Triathlon of lightweight block ciphers for the internet of things, IACR Cryptology ePrint Archive, 2015:209 (2015).

[78] Ravindran, R., Liu, X., Chakraborti, A., Zhang, X. and Wang, G.: Towards software defined icn based edge-cloud services, 2013 IEEE 2nd International Conference on Cloud Networking (CloudNet), pp.227235 (Nov. 2013).

[79] Baccelli, E., Mehlis, C., Hahm, O., Schmidt, T.C. and Wählisch, M.: Information centric networking in the iot: Experiments with ndn in the wild, arXiv preprint arXiv: 1406.6608 (Aug. 2014).

[80] Quevedo, J., Antunes, M., Corujo, D., Gomes, D. and Aguiar, R.L.: On the application of contextual iot service discovery in information centric networks, Computer Communications, pp.117-127 (Sep. 2016).

[81] Amadeo, M., Briante, O., Campolo, C., Molinaro, A. and Ruggeri, G.: Information-centric networking for $\mathrm{m} 2 \mathrm{~m}$ communications: Design and deployment, Computer Communications, pp.105-116 (Sep. 2016).

[82] Naito, K., Kamienoo, K., Nishio, T., Suzuki, H., Watanabe, A., Mori, K. and Kobayashi, H.: Proposal of seamless ip mobility schemes: Network traversal with mobility (ntmobile), IEEE GLOBECOM 2012 (Dec. 2012).

[83] Suzuki, H., Naito, K., Kamienoo, K., Hirose, T. and Watanabe, A. Ntmobile: New end-to-end communication architecture in ipv4 and ipv6 networks, ACM Mobicom 2013 (Sep. 2013). 


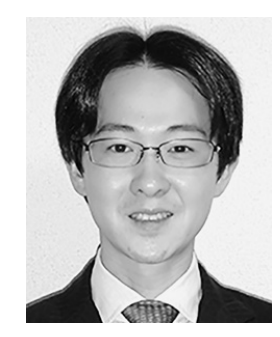

Katsuhiro Naito received his B.S. degree in Electronics Engineering from Keio University, Japan in 1999, and received his M.S. and Ph.D. degrees in Information Engineering from Nagoya University, Japan in 2001 and 2004, respectively. From 2004 to 2014, Dr. Naito was an assistant professor in the electrical and electronic engineering department of Mie university. He was a visiting scholar in the computer science department of University of California, Los Angeles (UCLA) in 2011. Since 2014, he has been an associate professor in the information science department of Aichi Institute of Technology. His research interests include 5G technologies, vehicular communication systems, Internet of Things (IoT) and Machine to Machine (M2M) systems, overlay networks, and network protocols and architectures. 